\title{
HYPERARITHMETICALLY ENCODABLE SETS
}

BY

ROBERT M. SOLOVAY

\begin{abstract}
We say that a set of integers, $A$, is hyperarithmetically (recursively) encodable, if every infinite set of integers $X$ contains an infinite subset $Y$ in which $A$ is hyperarithmetical (recursive). We show that the recursively encodable sets are precisely the hyperarithmetic sets. Let $\sigma$ be the closure ordinal of a universal $\Sigma_{1}^{1}$ inductive definition. Then $A$ is hyperarithmetically encodable iff it is constructible before stage $\sigma$.

We also prove an effective version of the Galvin-Prikry results that open sets, and more generally Borel sets, are Ramsey, and in the case of open sets prove that our improvement is optimal.
\end{abstract}

As usual, if $A$ and $B$ are subsets of $\omega, A \leqslant_{T} B$ (resp. $A \leqslant_{h} B$ ) means that $A$ is recursive (resp. hyperarithmetic) in $B$. Our paper studies the following notion of Jockusch and Soare: A set $A$ is hyperarithmetically (resp. recursively) encodable if for every infinite set $X \subseteq \omega$, there is an infinite set $Y \subseteq X$ such that $A \leqslant_{h} Y$ (resp. $A \leqslant_{T} Y$ ).

The following results about these notions are known from the literature:

(1) (Jockusch [6, Theorem 6.8]). Every hyperarithmetic set is recursively encodable.

(2) (Jockusch-Soare [7]). The complete $\Pi_{1}^{1}$ set, $\mathbf{O}$, of ordinal notations is not recursively encodable.

(3) (Jockusch-Soare [7]). Let $\alpha$ be the least recursively inaccessible ordinal. (I.e., $\alpha$ is the least admissible limit point of the class of admissible ordinals.) Let $L_{\alpha}$ be the collection of all sets constructible in less than $\alpha$ steps. Then if $A \in P(\omega) \cap L_{\alpha}, A$ is hyperarithmetically encodable.

The next result is an easy consequence of the techniques of Mathias [10]:

(4) Let $\beta_{0}$ be the least ordinal such that $L_{\beta_{0}}$ satisfies the axioms of $\mathbf{Z F}^{-}$. ( $\mathbf{Z F}^{-}$is $\mathbf{Z F}$ minus the power set axiom.) Then every hyperarithmetically encodable set lies in $L_{\beta_{0}}$ (and a fortiori is $\Delta_{2}^{1}$ ).

In this paper, we shall establish the following results:

(a) Every recursively encodable set is $\Delta_{1}^{1}$.

(b) Let $\sigma$ be the least $\Sigma_{1}^{1}$-reflecting ordinal. (Cf. [3].) Then every set in

Received by the editors February 14, 1975.

AMS (MOS) subject classifications (1970). Primary 02-00, 02F27, 02K30, 04A20; Secondary $02 \mathrm{~K} 05$.

Key words and phrases. Hyperarithmetically encodable set, Ramsey set, selective ultrafilter, inductive definition, Mathias forcing, Galvin-Prikry theorem.

- American Mathematical Society 1978 
$L_{\sigma} \cap P(\omega)$ is hyperarithmetically encodable.

(c) Conversely every hyperarithmetically encodable set lies in $L_{\sigma}$. Every arithmetically encodable set lies in $L_{\alpha}$.

(The ordinals we have introduced are ordered as follows: $\alpha<\sigma<\beta_{0}$. Moreover, each of $\sigma, \beta_{0}$ is "much larger" than its predecessors. For example $\sigma$ is recursively Mahlo and, a fortiori, is the oth recursively inaccessible ordinal.)

We shall also study the open set and Borel Ramsey problems. For example, let $B$ be a Borel subset of $2^{\omega}$. According to Galvin-Prikry [4], there is an infinite set $V \subseteq \omega$ such that either $V$ avoids $B$ (i.e., no infinite subset of $V$ lies in $B$ ) or $V$ lands in $B$ (i.e. every infinite subset of $V$ lies in $B$ ). In either case we say that $V$ is homogenous for $B$.

Suppose now that $U$ is an open set with recursive code. We shall prove the following results (which relativize straightforwardly):

(d) Suppose no $V$ avoids $U$. Then there is a hyperarithmetic $V$ that lands in $U$.

This has the following easy corollaries:

(e) There is a $V$ recursive in $\mathbf{O}$ (cf. (2) above), which is homogenous for $U$.

(f) Let $B$ be a Borel subset of $2^{\omega}$ with recursive code. Then there is a homogenous set for $B$ in $L_{\alpha^{\prime}}$ (Cf. (3) above.)

It follows from (1) together with the ideas of [14] that there is an open set with recursive code, $U$, which has no homogenous hyperarithmetic set $V$. We shall produce an explicit example:

(g) There is an open set, $U$, with recursive code, such that no $V$ lands in $U$. Moreover, if $V$ avoids $U$ then $V$ is neither $\Sigma_{1}^{1}$ nor $\Pi_{1}^{1}$.

We close this introduction with some historical remarks (which I learned from conversations with Jockusch).

First, say that a set of integers, $A$, is introreducible if it is recursive in each of its infinite subsets. It is easy to construct introreducible sets of any Turing degree [6], but it seems difficult to construct nontrivial examples of sets $A$ such that both $A$ and $\omega-A$ are introreducible. Indeed, Jockusch conjectures that if $A$ and its complement are both introreducible (call $A$ then bi-introreducible) then $A$ is recursive.

Note that if $A$ is bi-introreducible, $A$ is recursively encodable. Hence, by our result, (a), bi-introreducible sets are $\Delta_{1}^{1}$.

Jockusch also remarks that the uniform version of (a) is fairly easy. We say that $A$ is uniformly recursively encodable if there is an integer $e$ such that every infinite set $B$ contains an infinite subset $C$ such that $A$ is recursive in $C$ with the given Gödel number $e$.

Proposition (JoCKUSCH). $A$ is uniformly recursively encodable iff $A$ is $\Delta_{1}^{1}$.

Proof. $\leftarrow$ : This direction follows from Theorem 6.8 of [6]. 
$\rightarrow$ : Let $e$ be given by the definition. By the Galvin-Prikry theorem, $n \in A$ (resp. $n \notin A)$ iff $(\forall$ infinite sets $B)(\exists C$ infinite, $C \subseteq B)(\{e\} C(n)=1$ (resp. $\left.\{e\}^{C}(n)=0\right)$ ). Since $\{e\}^{C}$ is continuous in $C$, we have $n \in A($ resp. $n \notin A)$ iff $(\forall$ infinite sets $B)(\exists C$, finite, with $C \subseteq B)\left(\{e\}^{C}(n)=1\right.$ (resp. $\left.\left.=0\right)\right)$. Hence $A$ is $\Delta_{1}^{1}$.

\section{The effective Galvin-Prikry theorem.}

1.1. We begin with some heuristic comments on the proof. Our proof will be based on the notion of a selective ultrafilter. A nonprincipal ultrafilter $\mathcal{Q}$ on $\omega$ is selective if whenever $F$ maps $\omega^{[2]} \rightarrow 2$, then there is an $A \in \mathcal{Q}$ such that $F$ is constant on $A^{[2]}$. (Here $A^{[2]}$, for example, is the set of two element subsets of $A$.) Mathias showed that if $Q$ is selective and $B \subseteq 2^{\omega}$ is provably $\Delta_{2}^{1}$ (a fortiori, if $B$ is Borel), then there is a $V \subseteq \omega$ homogeneous for $B$, with $V \in$ थ.

Let $\omega_{1}$ denote the first nonrecursive ordinal. We shall work in $L_{\omega_{1}}$ with $थ$ a selective ultrafilter in the sense of $L_{\omega_{1}}$. We shall not quite be able to carry through Mathias' proof for the special case that $U$ is open. But by picking a थ with a certain technical property, which we call being decisive, we shall be able to show that if we fail to construct $V \in \mathcal{Q} \subseteq L_{\omega_{1}}$ which lands in $U$, then there is a $V \subseteq \omega$ (possibly not in $L_{\omega_{1}}$ ) which avoids $U$. The reader will not need to know Mathias' proof to understand our presentation.

1.2. Let $\lambda$ be an admissible ordinal such that in $L_{\lambda}$, every $\alpha<\lambda$ is countable. (For example, $\lambda$ could be any admissible ordinal $\leqslant \beta_{0}$.) Then a

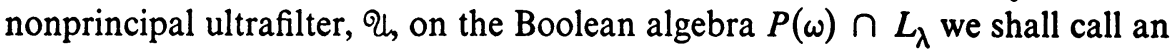

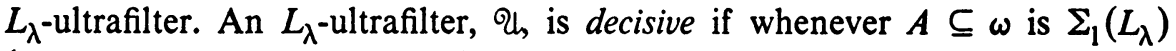
(parameters from $L_{\lambda}$ allowed) then there is an $X \in \mathcal{U}$ such that $X \cap A$ $=\varnothing$ or $X \subseteq A$.

Lemma. There is a decisive selective $L_{\lambda}$-ultrafilter of which is a $\Delta_{1}\left(L_{\lambda}\right)$ subset of $L_{\lambda}$.

Proof. We first outline the trivial construction of a $\Delta_{1}\left(L_{\lambda}\right)$ selective $L_{\lambda^{-}}$ ultrafilter. We then show how to modify the construction to get a decisive . $_{0}$

Let $\left\langle A_{\alpha} ; \alpha\langle\lambda\rangle\right.$ and $\left\langle F_{\alpha} ; \alpha\langle\lambda\rangle\right.$ be $\Delta_{1}\left(L_{\lambda}\right)$ enumerations of $P(\omega) \cap L_{\lambda}$ and $\left\{F \in L_{\lambda} \mid F:[\omega]^{2} \rightarrow 2\right\}$. For $\gamma$ a limit ordinal let $f_{\gamma}$ be the least bijection of $\omega$ onto $\gamma$. The map $\left\langle f_{\gamma} ; \gamma \in \operatorname{Lim} \cap \lambda\right\rangle$ is also clearly $\Delta_{1}\left(L_{\lambda}\right)$. We shall construct a $\Delta_{1}\left(L_{\lambda}\right)$ sequence, $\left\langle U_{\alpha}, \alpha\langle\lambda\rangle\right.$ with the following properties:

(1) If $\alpha<\beta, U_{\beta} \subset U_{\alpha}$ modulo finite sets. I.e., $U_{\beta}-U_{\alpha}$ is finite.

(2) If $\alpha<\lambda, U_{\alpha}$ is infinite.

(3) Either $U_{\alpha} \cap A_{\alpha}=\varnothing$ or $U_{\alpha} \subseteq A_{\alpha}$.

(4) $F_{\alpha}$ is constant on $U_{\alpha}^{[2]}$.

There is no difficulty meeting (1)-(4) in a step-by-step construction. Consider the case when $\alpha$ is a limit ordinal. Using $f_{\alpha}$ and induction hypothesis (1), we easily construct an infinite $U_{\alpha}^{*}$ such that for $\beta<\alpha, U_{\alpha}^{*}-U_{\beta}$ is finite. 
We may shrink $U_{\alpha}^{*}$ to $U_{\alpha}$ satisfying (2), (3), and (4) in a $\Delta_{1}\left(L_{\lambda}\right)$ fashion. (We use here the fact that the proof that there is a $U \subseteq U_{\alpha}^{*}$ satisfying (2) and (4) yields such a $U$ arithmetic in $U_{\alpha}^{*}, F_{\alpha}$.)

Let $U=\left\{A \subseteq \omega: A \cdot \in L_{\lambda}\right.$ and $U_{\gamma} \subseteq A$ for some $\left.\gamma<\lambda\right\}$. By (1)-(3), $U$ is a nonprincipal ultrafilter on $P(\omega) \cap L_{\lambda}$. By (4), $Q$ is selective. To determine if $A \in \mathcal{Q}$, simply find the $\alpha$ such that $A=A_{\alpha}$ and see if $U_{\alpha} \subseteq A_{\alpha}$ or not. Hence थ is $\Delta_{1}\left(L_{\lambda}\right)$.

It remains to see that $Q$ can be chosen decisive if the construction is done carefully. We distinguish two cases.

Case 1. Every $\Sigma_{1}\left(L_{\lambda}\right)$ subset of $\omega$ lies in $L_{\lambda}$. In this case, any $थ$ constructed as above is decisive.

Case 2. Otherwise.

Then there is a $\Delta_{1}\left(L_{\lambda}\right)$ function, $g$, which maps $\lambda 1-1$ into $\omega$. (Cf. [5], [12].) Let $\left\langle S_{\alpha} ; \alpha\langle\lambda\rangle\right.$ be one of the usual enumerations with repetitions of the $\Sigma_{1}\left(L_{\lambda}\right)$ subsets of $\omega$. For $\eta<\lambda$, let $S_{\alpha}^{\eta}$ be the subset of $S_{\alpha}$ listed by stage $\eta$.

Definition. The ordinal $\alpha$ is attackable at stage $\eta$ if the following conditions are met:

$(\alpha) \eta$ is a limit ordinal and $\alpha \leqslant \eta$.

(B) $S_{\alpha}^{\eta} \cap U_{\gamma}$ is infinite for each $\gamma<\eta$.

$(\gamma)$ For no $\gamma<\eta$ is $U_{\gamma} \subseteq S_{\alpha}^{\eta}$.

We shall impose the following extra condition on our construction:

(5) Suppose that $\eta$ is a limit ordinal, and that for some $\alpha \leqslant \eta, \alpha$ is attackable at stage $\eta$. Pick $\alpha_{0}$ attackable at stage $\eta$ so that $g\left(\alpha_{0}\right)$ is minimal. Then $U_{\eta} \subseteq S_{\alpha_{0}}^{\eta}$.

It is easy to see that the construction can be arranged so that (5) holds. We

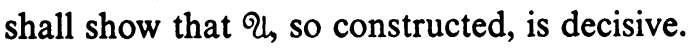

Suppose not. Pick $\alpha<\lambda$ such that $S_{\alpha} \cap U \neq \varnothing$ for every $U \in \mathcal{Q}$, but for no $\beta<\lambda$ is $U_{\beta} \subseteq S_{\alpha}$. We may assume that $\alpha$ was chosen so as to minimize $g(\alpha)$.

We show first that $\alpha$ is attackable at a closed cofinal set of stages less than $\lambda$.

(1) Let $\beta<\lambda$. Then $U_{\beta} \cap S_{\alpha}$ is infinite. (If not then $U_{\beta}-S_{\alpha} \in \mathcal{Q}$ and $S_{\alpha} \cap\left(U_{\beta}-S_{\alpha}\right)$ is empty. $)$

(2) There is an $\eta<\lambda$ such that $U_{\beta} \cap S_{\alpha}^{\eta}$ is infinite.

In fact, define $h: \omega \rightarrow \lambda$ in $\Delta_{1}\left(L_{\lambda}\right)$ by $h(n)$ is the least $\eta$ such that $U_{\beta} \cap S_{\alpha}^{\eta}$ has at least $n$ elements. Then if $\eta^{*}=\sup \{h(n): n<\omega\}$, then $\eta^{*}<\lambda$ since $\lambda$ is admissible, and $S_{\alpha}^{\eta^{*}} \cap U_{\beta}$ is infinite.

Now let $h_{1}(\beta)$ be the least $\eta$ such that $S_{\alpha}^{h_{1}(\beta)} \cap U_{\beta}$ is infinite. Then since $\lambda$ is admissible, standard arguments show that $K=\left\{\gamma<\lambda:(\forall \beta<\gamma) h_{1}(\beta)\right.$ $\langle\gamma\}$ is closed cofinal in $\lambda$. Clearly $\alpha$ is attackable at any stage $\eta$ in $K$ with $\eta \geqslant \alpha$.

Let us say that $\xi$ is attacked at stage $\eta$ if $\xi$ is attackable at stage $\eta$ and 
$U_{\eta} \subseteq S_{\xi}^{\eta}$. It follows from clause $(\gamma)$ of the definition of "attackable" that $\xi$ is attackable at no stage later than $\eta$.

Pick $\eta^{*}<\lambda$ so large that if $g(\gamma)<g(\alpha)$ and $\gamma$ is ever attacked, $\gamma$ will be attacked by stage $\eta^{*}$. (The set $\{\gamma \mid g(\gamma)<g(\alpha)\}$ is finite!) Pick $\eta<\lambda$ so that $\eta \geqslant \max \left(\alpha, \eta^{*}\right)$, and $\alpha$ is attackable at stage $\eta$. But then by (5) $\alpha$ is attacked at stage $\eta$ so $U_{\eta} \subseteq S_{\alpha}^{\eta} \subseteq S_{\alpha}$. But this contradicts our choice of $\alpha$.

1.3. From now on, $\mathcal{Q}$ is a fixed decisive nonprincipal selective $\Delta_{1}\left(L_{\omega_{1}}\right)$ ultrafilter on $P(\omega) \cap L_{\omega_{1}}$.

Let $s$ be a finite sequence of 0 's and 1's. Let $Y_{s}=\left\{f \in 2^{\omega} \mid s \subseteq f\right\}$. If $W \subseteq 2^{\omega}$ is open, let $C_{W}=\{s \mid s$ is a finite sequence of 0 's and l's and $\left.Y_{s} \subseteq W\right\}$. Via a Gödel numbering of the sequences, $C_{W}$ may be viewed as a subset of $\omega$. Clearly $C_{W}$ completely determines $W$ (and vice versa). We say that $W$ is recursive is $C_{W}$ is. We fix a recursive open set $W$ for the remainder of the proof.

1.4. Let $A$ be an infinite set, and $s$ a finite set. Say that $s<A$ if $\max (s)<\min (A)$. Let $A \mid s=\{x \in A \mid \max (s)<x\}$.

We now define for $s<A$ a certain ordinal invariant $\lambda(s, A)$.

(1) Let $s^{*}$ be the initial segment of the characteristic function of $s$ of length $\max (s)+1$. Then $\lambda(s, A)=0$ iff $s^{*} \in C_{W}$.

(2) Suppose that $\lambda(s, A) \neq \xi$ for all $\xi<\eta$. Suppose further that $(\forall y \in A)$ $(y>\max (s) \rightarrow \lambda(s \cup\{y\}, A \mid\{y\})<\eta \quad$ and $\eta=\sup \{\lambda(s \cup\{y\}, A \mid\{y\})$ $+1: y \in A \mid s\})$. Then we set $\lambda(s, A)=\eta$.

Lemma. (1) For $A \in L_{\omega_{1}}, \xi<\omega_{1}$, the relation $\lambda(s, A)=\xi$ is $\Delta_{1}\left(L_{\omega_{1}}\right)$.

(2) For $A \in L_{\omega_{1}}, \lambda(s, A)<\omega_{1}$.

(3) If $\lambda(s, A)<\omega_{1}($ so $s<A)$, then for every $B \subset \omega$ such that $s \subseteq B \subseteq s \cup$ $A$, and $B$ is infinite, we have $B \in W$.

(4) Let $B \subseteq A, B$ infinite. Then $\lambda(s, B) \leqslant \lambda(s, A)$.

Proof. (1) We shall use the techniques of meta-recursion theory. (Cf. [12], for example.) It is easy to construct a $\Sigma_{1}\left(L_{\lambda}\right)$ partial function $f(A, s, \xi, \varepsilon)$ with the following property. Suppose $\varepsilon$ is the index of a partial recursive function of three variables. Suppose that for all $\eta<\xi$, all infinite $B \in L_{\omega_{1}} \cap P(\omega)$ and all finite subsets of $\omega, t$, with $t<B$, we have $\{\varepsilon\}(B, t, \eta)$ is defined and equals 0 or 1. Finally, assume that $\{\varepsilon\}(B, t, \eta)=0$ under these conditions iff $\lambda(t, B)$ $=\eta$. Then $f(A, s, \xi, \varepsilon)$ is defined and equals 0 or 1 . Moreover $f(A, s, \xi, \varepsilon)=0$ iff $\lambda(s, A)=\xi$.

We now invoke the recursion theorem to find $\varepsilon_{0}$ so that

$$
\left\{\varepsilon_{0}\right\}(A, s, \xi) \simeq f\left(A, s, \xi, \varepsilon_{0}\right) .
$$

One checks by induction on $\xi$ that $\lambda(s, A)=\xi$ (resp. $\lambda(s, A) \neq \xi$ ) iff $\left\{\varepsilon_{0}\right\}(A, s, \xi)=0($ resp. $=1)$. 
(2) If for some $A \in L_{\omega_{1}}, \lambda(s, A) \geqslant \omega_{1}$, pick $s$ and $A$ so that $\lambda(s, A)$ is minimal in $\left[\omega_{1}, \infty\right)$. Then $\lambda(s, A)=\omega_{1}$, and using (1), this contradicts the admissibility of $\omega_{1}$.

(3) Let $s$ have $k$ elements. Let $s_{n}$ be the first $n+k$ elements of $B \cup s$. By construction of the invariant $\lambda(\cdot, \cdot)$, if $\infty>\lambda\left(s_{n}, A \mid s_{n}\right)>0$, then $\lambda\left(s_{n+1}\right.$, $\left.A \mid s_{n+1}\right)<\lambda\left(s_{n}, A \mid s_{n}\right)$. Hence for some $n, \lambda\left(s_{n}, A \mid s_{n}\right)=0$. But this shows $B \in W$.

(4) The proof is by induction on $\lambda(s, A)$ and is straightforward.

1.5. We quote for future reference two lemmas.

LEMMA 1 ( $\Sigma_{1}$-choice). Let $\lambda$ be an admissible ordinal. Let $S\left(x_{1}, y\right)$ be a $\Sigma_{1}\left(L_{\lambda}\right)$ relation. Suppose $a \in L_{\lambda}$. Suppose $(\forall x \in a)(\exists y) S(x, y)$. Then there is an $f \in L_{\lambda}$ with domain $(f)=a$ and $(\forall x \in a) S(x, f(x))$.

ProOF (WeLL +KNOWN). Write $S(x, y)$ as $(\exists z) T(x, y, z)$ with $T \Sigma_{0}$. Let $g(x)$ be the least $\langle y, z\rangle \in L_{\lambda}$ such that $T(x, y, z)$. Then $g\left\lceil a \in L_{\lambda}\right.$ since $L_{\lambda}$ is admissible. Let $f(x)=\pi(g(x))$ where $\pi(\langle y, z\rangle)=y$.

LEMMA 2. Let $थ$ be our fixed selective ultrafilter on $L_{\omega_{1}}$. Let $\left\langle U_{n} ; n \in \omega\right\rangle$ be a sequence in $L_{\omega_{1}}$ of members of $\vartheta_{0}$. Then there is a $U \in \mathcal{U}$ such that for all $n \in U, U \mid\{n\} \subseteq U_{n}$.

Proof. Define $F: \omega^{[2]} \rightarrow 2: F(\{m, n\})=1$ iff $n \in U_{m}$ (for $\left.n>m\right)$. Let $U$ $\in \mathcal{Q}$ be $F$-homogeneous. Pick $m \in U$ and $n>m$ in $U \cap U_{m}$. Then $F(\{m$, $n\})=1$. So $F$ is $\equiv 1$ on $U^{[2]}$. Thus $U$ satisfies the demands of the lemma.

1.6. Definition. $\lambda(s)$ is defined iff $\lambda(s, U)$ is defined for some $U \in$ U. If so, $\lambda(s)=\inf \{\lambda(s, U): U \in \mathcal{Q}\}$.

Lemma. Suppose $\lambda(s)$ is not defined. Then for some $U \in \mathcal{Q}$, with $U>s$, $\lambda(s \cup\{x\})$ is not defined for all $x \in U$.

Proof. Let $A=\{x \mid x>\max (s)$ and $(\exists U \in \mathcal{Q}) \lambda(s \cup\{x\}, U)$ is defined $\}$. Then $A$ is $\Sigma_{1}\left(L_{\omega_{1}}\right)$. Since $Q$ is decisive, there is a $U \in \mathcal{Q}$ such that $U \cap A$ $=\varnothing$ or $U \subseteq A$. If $U \cap A=\varnothing$ the lemma is proved. We assume $U \subseteq A$ and show that contrary to our hypothesis, $\lambda(s)$ is defined.

By the $\Sigma_{1}$ axiom of choice (Lemma 1.5.1) there is, in $L_{\omega_{1}}$, a sequence $\left\langle U_{n} ; n \in U\right\rangle$ such that $\lambda\left(s \cup\{n\}, U_{n} \mid\{n\}\right)$ is defined and $U_{n} \in \mathcal{Q}$ for each $n \in U$.

By Lemma 1.5.2, we can find $U^{*} \in \mathcal{Q}$ such that (1) $U^{*} \subseteq U$, (2) $n \in U^{*}$ $\rightarrow U^{*} \mid\{n\} \subseteq U_{n}$. But then $\lambda\left(s \cup\{n\}, U^{*} \mid\{n\}\right)$ is defined for each $n \in U^{*} \mid s$. It follows that $\lambda\left(s, U^{*}\right)$ is defined. Since $U^{*} \in \mathcal{Q}, \lambda(s)$ is defined, contrary to our hypothesis. Thus the assumption $U \subseteq A$ is absurd.

1.7. Lemma. Let $\lambda(\varnothing)$ be undefined. Then there is an infinite set $B$ such that $\lambda(s)$ is undefined for each finite subset $s$ of $B$. 
Proof. We define an increasing sequence of finite sets $B_{n}$ so that (1) $B_{n}$ has $n$ elements and (2) $\lambda(s)$ is not defined for each $s \subseteq B_{n}$. If we can do this, the desired $B$ is just the union of the $B_{n}$ 's.

For $B_{0}$, we simply take $\varnothing$. Suppose $B_{n}$ is defined satisfying (1) and (2). Let $s_{1}, \ldots, s_{2^{n}}$ be an enumeration of the finite subsets of $B_{n}$.

Let $W_{j} \in \mathcal{Q}$ with $W_{j}>s_{j}$ and $\lambda\left(s_{j} \cup\{x\}\right)$ undefined for each $x \in W_{j}$. (By Lemma 1.6, such a $W_{j}$ exists.) Then $\cap_{j=1}^{2 n} W_{j}$ is in $U$. Pick $x$ in this intersection greater than $\max \left(B_{n}\right)$ and set $B_{n+1}=B_{n} \cup\{x\}$. Then (1) and (2) hold for $B_{n+1}$.

1.8. THEOREM. Let $W$ be an open subset of $2^{\omega}$. Let $C_{W}$ be the canonical code for $W$. Then either there is an infinite set $V$ that avoids $W$ or there is an infinite set $V$ hyperarithmetic in $C_{W}$ which lands in $W$.

Proof. Assume first that $C_{W}$ is recursive. Let $Q$ be as above. Suppose that $\lambda(\varnothing)$ is undefined, and let $B$ be as in Lemma 1.7. We show that $B$ avoids $W$. Suppose not. Let $C \subseteq B, C$ infinite such that $C \in W$. Since $W$ is open we can find $n$ such that if $D \cap\{0, \ldots, n\}=C \cap\{0, \ldots, n\}$ then $D \in W$. Since $C$ is infinite, we may assume that $n \in C$ (increasing $n$ if necessary). Let $s$ $=C \cap\{0, \ldots, n\}$. Then $\lambda(s)=0$, contrary to our assumptions on $B$.

If $\lambda(\varnothing)$ is defined, pick $U \in \mathcal{Q}$ so that $\lambda(\varnothing, U)$ is defined. By (3) of Lemma 1.4, $U$ lands in $W$. And of course $U \in \mathcal{Q} \subseteq L_{\omega_{1}}$ so $U$ is hyperarithmetic. This proves the special case when $C_{W}$ is recursive.

The proof relativizes straightforwardly to give Theorem 1.8 in general.

1.9. Corollary. Let $W$ be an open set. Let $O^{W}$ be a complete $\Pi_{1}^{1}\left(C_{W}\right)$ subset of $\omega$. Then there is an infinite $V \subseteq \omega$ homogeneous for $W$ which is recursive in $O^{W}$.

PRoof. If no infinite $V \subseteq \omega$ avoids $W$, this is clear by Theorem 1.8 . Suppose then that there is a $V$ that avoids $W$. Let $\delta=\{V \subseteq \omega \mid V$ is infinite and avoids $W$ \}. It suffices, by the Kleene basis theorem [11], to show that $\delta$ is $\Sigma_{1}^{1}\left(C_{W}\right)$.

But $V \in \delta$ iff $V$ is infinite and $(\forall s \subseteq V)(s$ finite $\rightarrow \lambda(s, \omega / s) \neq 0)$. This shows that $\delta$ is even $\Pi_{2}^{0}\left(C_{W}\right)$.

1.10. Corollary. Let $B$ be a Borel subset of $2^{\omega}$ with code $\beta$. (Cf. $[15$, Chapter II].) Let $\lambda$ be recursively inaccessible in $\beta$. Then there is a $Y \in L_{\lambda}[\beta]$ which is homogeneous for $B$.

Proof. Let $M=\left\langle L_{\lambda}[\beta] ; \varepsilon, \beta\right\rangle$. By Corollary 1.9 , if $W$ is an open set, with $C_{W}$ in $M$, then there is a homogeneous set $V$ for $W$ in $M$. Moreover $V$ can be taken to be a $\Delta_{1}^{1}(M)$ function of $W$. Indeed, since $\lambda$ is recursively inaccessible in $\beta$, we can in $\Delta_{1}^{l}(M)$ fashion determine the truth of $\Pi_{1}^{1}$ sentences with 
parameters in $M$. To find $V$, simply search through those subsets of $\omega$ recursive in $O^{W}$ checking whether they are homogeneous for $W$.

It is now straightforward to carry out the usual Galvin-Prikry proof of the existence of a $Y$ homogeneous for $B$ inside $M$, using the effective solution of the open Ramsey problem just noted.

2. Recursively encodable sets.

2.1. In this section, we shall show that every recursively encodable set is $\Delta_{1}^{1}$. Our motivation is the classical result that a real that appears in $M[x]$ for every $x$ generic over the countable transitive model of ZFC, $M$, must lie in $M$. Roughly speaking, we replace $M$ by $L_{\omega_{1}}$, and the usual Cohen conditions by the Mathias type conditions $\langle s, U\rangle$ where $s$ is a finite subset of $\omega, U \in \mathcal{Q}$, and $s<U$. However, the usual notion of "generic" will be replaced by a slightly different notion adapted to our present purpose.

Definition. A system of restraints is a collection $R$ of ordered pairs $\langle s, U\rangle$ with the following properties:

(1) If $\langle s, U\rangle \in R$, then $s$ is a finite subset of $\omega, U \in \mathcal{U}$ (the ultrafilter on $L_{\omega_{1}}$ constructed in Lemma 1.2), and $s<U$.

(2) For each finite set $s \subseteq \omega, s$ is the first member of only finitely many pairs in $R$.

Definition. A set $X$ meets the system of restraints $R$, if $X$ is infinite and if whenever $s \cup\{x\} \subseteq X, x>\max (s)$, and $\langle s, U\rangle \in R$, then $x \in U$.

LEMMA. Let $R$ be a system of restraints. Then there is an infinite set $X$ meeting $R$. If $X$ meets $R$ and $Y \subseteq X, Y$ infinite, then $Y$ meets $R$.

Proof. The first claim is proved along the lines of Lemma 1.7. The second is clear from the definitions.

2.2. We now wish to express the results of $\S 1$ in the language of systems of restraint. If $C$ is the code for an open set, let $\lambda(s, A ; C)$ be the invariant introduced in $\$ 1.4$. (We remark parenthetically that the set of codes for open sets is easily seen to be $\Pi_{1}^{0}$.)

LEMMA. Let $W$ be an open set with a hyperarithmetic canonical code, $C$. Let $s$ be a finite set of integers. Then either $\lambda(s, U)$ is defined for some $U \in \mathcal{U}$ (in which case every infinite $X$ with $s \subseteq X \subseteq s \cup U$ lies in $W$ ) or else there is a system of restraints $R$ such that:

(1) If $X$ meets $R$ and $s$ is an initial segment of $X$, then $X \notin W$.

(2) If $\langle t, U\rangle \in R$, then $t$ is an end-extension of s. (I.e. $s=t \cap\{0, \ldots, n\}$ for some n.)

Proof. If $\lambda(s ; C)$ is defined, the lemma is clear. If not for each endextension $t$ of $s$ such that $\lambda(t ; C)$ is undefined, pick $U_{t} \in \mathcal{Q}$ with $t$ $<U_{t}$ and $\lambda(t \cup\{x\})$ undefined for each $x \in U_{t}$. (Cf. Lemma 1.6.) Then if 
$R=\left\{\left\langle t, U_{t}\right\rangle: U_{t}\right.$ is defined $\}$, then $R$ meets the requirements of the lemma.

2.3. Theorem. Let $A \subseteq \omega, A$ not hyperarithmetic. Then $A$ is not recursively encodable.

Proof. In view of Lemma 2.1, it suffices to produce a system of restraints $R$ such that if $X$ meets $R$, then $A$ is not recursive in $X$.

Let us say that a system of restraints $R$ has height $\geqslant n$ if whenever $\langle s, U\rangle \in R$, then $s$ has at least $n$ elements. The definition of "system of restraints" is so arranged that if for each $n, R_{n}$ is a system of restraints of height $\geqslant n$, then $R=\cup_{n \in \omega} R_{n}$ is a system of restraints. Moreover if $X$ meets $R, X$ meets each $R_{n}$. Hence it suffices to prove for each integer $n$, there is a system of restraints of height $\geqslant n, R_{n}$, such that if $X$ meets $R_{n}, A$ is not recursive in $X$ with Gödel number $n$.

2.4. We are going to make another preliminary reduction. Say that a system of restraints, $R$, is centered at $s$ if each $t$ in domain $(R)$ is an end-extension of $s$. We shall now show that the theorem follows from the following:

LEMMA. Let $s$ be a finite subset of $\omega$ with $n$ elements. Then there is a system of restraints, $R_{s}$, centered at $s$, such that if $X$ meets $R_{s}$ and $s$ is an initial segment of $X$, then $A$ is not recursive in $X$ with Gödel number $n$.

Indeed, granted the lemma, let $R_{n}=\bigcup\left\{R_{s}: s \subseteq \omega\right.$ and $s$ has $n$ elements $\}$. Then $R_{n}$ is a system of restraints of height $\geqslant n$, and if $X$ meets $R_{n}, A$ is not recursive in $X$ with Gödel number $n$.

2.5. We turn to the proof of Lemma 2.4. We fix $n$ and $s$. Define a sequence of recursive open sets $\left\langle W_{j, \varepsilon} ; j<\omega, \varepsilon<2\right\rangle$ as follows.

$$
W_{j, \varepsilon}=\left\{X \subseteq \omega \mid\{n\}^{X}(j)=\varepsilon\right\} .
$$

We let $\lambda(s, U ; j, \varepsilon)$ be the invariant of $\S 1.3$ relative to the set $W_{j, \varepsilon}$. Similarly for $\lambda(s ; j, \varepsilon)$.

Case 1. For some $j, \lambda(s ; j, 0)$ and $\lambda(s ; j, 1)$ are undefined.

By Lemma 2.2, there is a system of restraints centered at $s, R_{s}$, such that if $X$ meets $R_{s}$ and $s$ is an initial segment of $X$, then $\{n\}^{X}(j)$, if defined, is unequal to 0 or 1 . It follows that (the characteristic function of) $A$ is not recursive in $X$ with Gödel number $n$.

Thus in this case, Lemma 2.4 is proved. Henceforth, we assume Case 1 does not hold.

Lemma. $\lambda(s ; j, \varepsilon)$ is defined for at most one $\varepsilon<2$.

Proof. Suppose not. Pick $U_{0}, U_{1}$ such that $\lambda\left(s, U_{0} ; j, 0\right)$ and $\lambda\left(s, U_{1} ; j, 1\right)$ are defined and $U_{0}, U_{1}$ are in $\vartheta_{\text {. Let }} U=U_{0} \cap U_{1}$. Then $\lambda(s, U ; j, 0)$ and $\lambda(s, U ; j, 1)$ are defined. 
It follows that $s \cup U \in W_{j, 0} \cap W_{j, 1}=\varnothing$, which is absurd.

2.6. Let $B_{\varepsilon}=\{n \mid \lambda(s ; n, \varepsilon)$ is defined $\}$. Clearly $B_{\varepsilon}$ is $\Sigma_{1}\left(L_{\omega_{1}}\right)$. Our assumption that Case 1 does not hold and Lemma 2.5 entail that $B_{1}=\omega-B_{0}$. Hence $B_{1}$ is hyperarithmetic, and so unequal to $A$.

Pick $j$ so that $j \in B_{1}$ iff $j \notin A$. By Lemma 2.2 , we can find $U_{s}$ so that (1) $U_{s} \in \mathcal{Q}$, (2) $U_{s}>s$, (3) if $s \subseteq X \subseteq s \cup U_{s}$, then $\{n\}^{X}(j)=1-\chi_{A}(j)$. (This is possible since $j \in B_{0} \cup B_{1}$ !) Then if $R_{s}=\left\{\left\langle s, U_{s}\right\rangle\right\}, R_{s}$ meets the requirements of Lemma 2.4. This completes the proof of Theorem 2.3.

3. Hyperarithmetic sets do not solve the open Ramsey problem.

3.1. THEOREM. There is an open set $W$ with recursive code such that

(1) No infinite set lands in $W$.

(2) If $V \subseteq \omega$ avoids $W$, and $V$ is infinite then $V$ is neither $\Sigma_{1}^{1}$ or $\Pi_{1}^{1}$.

Proof. Our starting point is the following well-known lemma which plays a crucial role in the paper [7].

LEMMA. There is a recursive set of finite sequences of integers, $T$, with the following properties:

(1) $T$ is a tree. I.e., if $s \in T$, and $t$ is an initial segment of $s$, then $t \in T$.

(2) $T$ has branches. I.e., there is an $f: \omega \rightarrow \omega$ such that each finite initial segment of $f$ lies in $T$.

(3) $T$ has no hyperarithmetic branch.

3.2. We define an open set $W$ as follows. Let $X \subseteq \omega$. $X$ is in $W$ if for some $n \in \omega$ and $\left\langle x_{0}, \ldots, x_{n-1}\right\rangle$ we have:

(1) $\left\{x_{0}, \ldots, x_{n-1}\right\}$ are the first $n$ members of $X$.

(2) If $t_{i} \leqslant x_{i}$ for $0 \leqslant i \leqslant n-1$, then $\left\langle t_{0}, \ldots, t_{n-1}\right\rangle \notin T$.

It is easy to check that $W$ is recursive since $T$ is.

Lemma. Let $X \subseteq \omega, X$ infinite. Then there is a $Y \subseteq X$ such that $Y$ is infinite and $Y \notin W$.

Proof. The principal function, $p_{X}$, of an infinite set $X$ is the function that enumerates $X$ without repetitions in increasing order.

Let now $f$ be a branch of $T$. Pick $Y \subseteq X, Y$ infinite, such that $p_{Y}(n) \geqslant f(n)$, for all $n \in \omega$. Then $Y \notin W$.

3.3. The following lemma is also a key tool of [7].

LEMMA. Let $T$ be a tree of finite sequences of integers. Let $g: \omega \rightarrow \omega$. Suppose that $T$ has a branch $f$ such that $f(n) \leqslant g(n)$ for all but finitely many $n$. Then $T$ has a branch $f$ arithmetic in $T, g$.

COROllary. Let $V$ be infinite and avoid $W$. Then $V$ is not $\Pi_{1}^{1}$. 
Proof. Suppose $V$ is infinite, $\Pi_{1}^{1}$, and avoids $W$. Then $V$ contains an infinite hyperarithmetic subset, $V^{*}$, with $V^{*} \notin W$. By König's lemma, $T$ has a branch $f$ such that $f(n)<p_{\nu^{*}}(n)$ for all $n$. (Cf. the definition of $W$ !) Hence by the lemma we may suppose that $f$ is arithmetic in $V^{*}, T$ and so is hyperarithmetic. But $T$ has no hyperarithmetic branch.

3.4. For future use, we isolate out part of the proof of Lemma 3.3.

LEMma. Let $T$ be a tree of finite sequences. Let $g: \omega \rightarrow \omega$. Suppose that for each $n$, there is an n-tuple $\left\langle x_{0}, \ldots, x_{n-1}\right\rangle \in T$ with $x_{i} \leqslant g(i)$ for $0 \leqslant i<n$. Then $T$ has a branch $f$, arithmetic in $T$ and $g$, with $f(n) \leqslant g(n)$ for all $n$.

3.5. We turn now to the proof that no $\Sigma_{1}^{1}$ set, $V$, avoids $W$.

Lemma. Let $V$ be $\Sigma_{1}^{1}$. There is a function $f: \omega_{1} \times \omega \rightarrow \omega$ with the following properties:

(1) $f$ is $\Delta_{1}\left(L_{\omega_{1}}\right)$;

(2) For fixed $n, f(\alpha, n)$ is weakly monotone increasing in $\alpha$;

(3) For each $n<\omega$, there is a $\sigma_{n}<\omega_{1}$ such that for $\sigma_{n} \leqslant \alpha<\omega_{1}, f(\alpha, n)$ $=p_{V}(n)$.

Proof. Since $V$ is $\Sigma_{1}^{1}, \omega-V$ is the range of some $\Delta_{1}\left(L_{\omega_{1}}\right)$ function $g: \omega \rightarrow \omega$. Let $V_{\alpha}=\omega-\{g(\beta): \beta<\alpha\}$. Let $f(\alpha, n)=p_{V_{\alpha}}(n)$. Then the properties of $f$ asserted in the lemma are easy to check.

3.5. We now invoke the $\Sigma_{1}$ axiom of choice, and get a sequence $\left\langle s_{n} ; n \in \omega\right\rangle$ in $L_{\omega_{1}}$ such that

(1) $s_{n}$ is a sequence of length $n, s_{n} \in T$.

(2) For some $\alpha<\omega_{1}, x_{n}(i)<f(\alpha, i)$ for $0<i<n$.

We define a function $f^{*}$ in $L_{\omega_{1}}$ by $f^{*}(n)=\sup \left\{s_{m}(n): m>n\right\}$. Note that the sup exists since $s_{m}(n) \leqslant f(\alpha, n) \leqslant p_{V}(u)$. By Lemma 3.4, there is a branch arithmetic in $f^{*}$. But this is absurd since $T$ has no hyperarithmetic branches. This completes the proof of Theorem 3.1.

3.6. The following variant of the argument of $\S 3.5$ will be useful in $\S 4$.

Lemma. Let $X \subseteq \omega$. Let $M=L_{\omega_{1} X}[X]$. Let $T$ be a tree of finite sequences of integers which is $\Sigma_{1}(M)$. Suppose that $T$ has a branch $f$ such that $f(n)<p_{X}(n)$ for all $n \in \omega$. Then $T$ has a branch in $M$.

Proof. By $\Sigma_{1}$ choice, we may find a function $\left\langle s_{n} ; n \in \omega\right\rangle$ in $M$ such that

(1) $s_{n}$ is a sequence of length $n$;

(2) $s_{n} \in T$;

(3) $s_{n}(i) \leqslant p_{X}(i)$ for $i<n$.

Let $T^{*}=\left\{s \mid\right.$ For some $n, s$ is an initial segment of $\left.s_{n}\right\}$. Then $T^{*}$ is a tree, $T^{*}$ has arbitrarily long members, and for $s \in T^{*}$, and $n<$ length $(s), s(n)<$ $p_{X}(n)$. By König's lemma and Lemma 3.3, $T^{*}$ (and hence $T$ ) has a branch $g$ arithmetic in $T^{*}, p_{X}$. But then $g \in M$. 
4. An improved lower bound for the class of hyperarithmetically encodable sets.

4.1. If $f$ and $g$ are in ${ }^{\omega} \omega$, we say that $g$ dominates $f$ if $f(n) \leqslant g(n)$ for all but finitely many $n$. We say that a set $A$ is strongly hyp. encodable if there is a function $f_{A} \in{ }^{\omega} \omega$ such that whenever $g \in{ }^{\omega} \omega$ dominates $f_{A}$, then $A<_{h} g$. Jockusch and Soare prove that if $A \in L_{\alpha}$ (cf. (3) of the introduction), then $A$ is strongly hyp. encodable. It is easy to see that if $A$ is strongly hyp. encodable, $A$ is hyp. encodable.

If $\gamma$ is a countable ordinal, then we say that $\gamma$ is hyp. encodable if $\gamma<\omega_{1}^{A}$ for some hyp. encodable $A$. There is an analogous notion of $\gamma$ being strongly hyp. encodable.

4.2. Let $\gamma$ be an ordinal which we wish to prove strongly hyp. encodable. The following lemma gives a sufficient condition. In the work of Jockusch and Soare, the analogous condition is that $\gamma$ is not recursively inaccessible and all $\eta<\gamma$ are strongly hyp. encodable. Our improvement comes from working with trees that are $\Sigma_{1}\left(L_{\gamma}\right)$ rather than trees in $L_{\gamma}$.

LEMMA. Let $\gamma$ be a countable ordinal. We suppose that each $\eta<\gamma$ is strongly hyp. encodable. If $\gamma$ is admissible, we suppose that there is a tree $T$ of finite sequences of integers with the following properties:

(1) $T$ is $\Sigma_{1}\left(L_{\gamma}\right)$;

(2) $\gamma<\omega_{1}^{f}$ for every branch $f$ of $T$;

(3) $T$ has branches.

Assume finally that $\gamma<\beta_{0}$, the least ordinal such that $\left.L_{\beta_{0}}=\mathbf{Z F}{ }^{-1}\right)$. Then $\gamma$ is strongly hyp. encodable.

Proof. Let $h$ map $\omega$ onto $\gamma$. For each $n$ select a subset $A_{n}$ of $\omega$ and a function $f_{n} \in{ }^{\omega} \omega$, so that $h(n)<\omega_{1}^{A_{n}}$ and $A_{n} \leqslant n g$ whenever $g$ dominates $f_{n}$. If $\gamma$ is admissible, let $f^{*}$ be a branch through the $T$ provided for by our hypothesis. Define $f \in{ }^{\omega} \omega$ by

$$
f(n)=\max \left(f^{*}(n), f_{0}(n), \ldots, f_{n}(n)\right) .
$$

We shall show that there is a set $A$ such that $\gamma<\omega_{1}^{A}$ and $A \leqslant_{h} g$ whenever $g$ dominates $f$.

We may assume $\gamma$ is primitive recursively closed since otherwise the lemma is trivial. (Take $\eta$ the largest p.r. closed ordinal $<\gamma$ and observe that if $\eta$ is strongly hyp. encodable, so is $\gamma$.) We take $A$ to be the set of Gödel numbers of true sentences of the structure $\left\langle L_{\gamma} ; \varepsilon\right\rangle$. Since $\gamma<\beta_{0}, \gamma<\omega_{1}^{A}$.

Let now $g \in{ }^{\omega} \omega$ such that $g$ dominates $f$. A fortiori, $g$ dominates $f_{n}$ for each

(1) This hypothesis will be removed in $\$ 5$ where we prove from our other hypothesis that $\gamma<\sigma<\beta_{0}$. 
$n$. Hence $h(n)<\omega_{1}^{g}$ for each $n$, and so $\gamma \leqslant \omega_{1}^{g}$. If $\gamma$ is not admissible, then $\gamma<\omega_{1}^{g}$. But if $\gamma<\omega_{1}^{g}$, then $A=\operatorname{Tr}\left(\left\langle L_{\gamma} ; \varepsilon\right\rangle\right)$ is clearly hyp. in $g$.

Suppose then that $\gamma=\omega_{1}^{g}$. We derive a contradiction. The tree $T$ is $\Sigma_{1}\left(L_{\gamma}\right)$ and a fortiori is $\Sigma_{1}\left(L_{\gamma}[g]\right)$. Also $g$ dominates the branch $f^{*}$ of $T$. By Lemma 3.6, $L_{\gamma}[g]$ contains a branch of $T$. But $\gamma$ is recursive in each branch of $T$, and by our assumption that $\gamma=\omega_{1}^{g}$, the structure $L_{\gamma}[g]$ is admissible. So we must have $\gamma<\omega_{1}^{g}$ and so $A \leqslant_{h} g$.

4.3. We wish to translate the information given by Lemma 4.2 into more familiar terms. The following concept is taken from Aczel-Richter [3].

Definition. An admissible ordinal $\gamma$ is $\Sigma_{1}^{1}$-reflecting if whenever $\phi$ is a $\Sigma_{1}^{1}$ sentence (with parameters from $L_{\gamma}$ ) such that $L_{\gamma} \vDash \phi$ then for some admissible ordinal $\delta<\gamma, L_{\delta} \vDash \phi\left({ }^{2}\right)$.

LeMma. Let $\gamma$ be an admissible ordinal with $\gamma<\beta\left({ }^{3}\right)$ then the following are equivalent:

(1) $\gamma$ is not $\Sigma_{1}^{1}$-reflecting.

(2) There is a tree $T$ with the properties stated in the hypothesis of Lemma 4.2.

Proof. Assume first that $T$ is a tree with the properties asserted in Lemma 4.2 and that $\gamma$ is $\Sigma_{1}^{1}$-reflecting. We shall derive a contradiction.

Note first that $\gamma$ is a limit of admissible ordinals. (This is immediate from the definition of " $\Sigma_{1}^{1}$-reflecting".) Using the $\Sigma_{1}\left(L_{\gamma}\right)$ definition of $T$, let $\phi$ be a $\Sigma_{1}^{1}$ sentence asserting that $T$ has a branch. Let $\delta<\gamma$ such that $L_{\delta} \vDash \phi$. Let $T^{*}$ be the tree defined in $L_{\delta}$ by the given $\Sigma_{1}$ definition of $T$. Then $T^{*}$ $\subseteq T$ since $\Sigma_{1}$ sentences true in $L_{\delta}$ remain true in $L_{\gamma}$. Since $L_{\delta} \vDash \phi, T^{*}$ has a branch. Since $T^{*} \in L_{\gamma}$, and $\gamma$ is a limit of admissibles, $T^{*}$ has a branch $f$ in $L_{\gamma}$. By our assumption, $\gamma<\omega_{1}^{f}$. But this contradicts the admissibility of $\gamma$. (This argument is presumably due to Aczel; cf. [2].)

We now assume that $\gamma$ is an admissible ordinal $<\beta$ which is not $\Sigma_{1}^{1}$ reflecting. We let $(\exists X) \phi(X)$ be a $\Sigma_{1}^{1}$ statement such that $L_{\gamma} \vDash(\exists X) \phi(X)$ but if $\delta<\gamma$ is admissible, $L_{\delta} \vDash \neg(\exists X) \phi(X)$. (Here $\phi$ is first order with parameters from $L_{\gamma}$, and in the preceding sentence, it is understood that only $\delta$ 's such that $L_{\delta}$ contains the parameters of $\phi$ are considered.) Let $\delta_{0}<\gamma$ be a primitive recursively closed ordinal so that all parameters appearing in $\phi$ appear in $L_{\delta_{0}}$. Let $h: \gamma \rightarrow \omega$ be a $\Sigma_{1}\left(L_{\gamma}\right)$ map of $\gamma 1-1$ into $\omega$. ( $h$ exists since $\gamma<\beta$.) Let $g \in L_{\gamma}$ map $\delta_{0} 1-1$ onto $\omega$.

Instead of giving a detailed description of $T$ we describe it heuristically. A branch of $T, f$, will determine a model of $K P\left({ }^{4}\right)+V=L, M$, with $|M|=\omega$.

(2) We would get the same notion if we deleted the work "admissible".

(3) Here $\beta$ is the least ordinal not projectable into $\omega$.

(4) $K P$ is the first order theory whose well-founded models are the admissible sets. 
Also $f$ will encode a subset $X$ of $\omega$. In addition, $f$ will encode an order preserving map of the ordinals of $M$ into $\gamma$. Thus there is an isomorphism $\psi: M \cong L_{\delta}$ for some admissible $\delta \leqslant \gamma$. We shall arrange finally that $\delta>\delta_{0}$ and that $\left\langle L_{\delta}, \psi[X]\right\rangle \vDash \phi(\psi[X])$. The construction of a $T$ whose branches are as stated is relatively straightforward (cf. the following paragraph) and does not use the fact that $\phi$ does not reflect down. It will be easy to see that $T$ is $\Sigma_{1}\left(L_{\gamma}\right)$. Moreover since $L_{\gamma} \vDash(\exists X) \phi(X), T$ will have branches.

Let then $f$ be a branch through $T$. Then recursive in $f$ is an admissible ordinal $\delta$ such that $L_{\delta} \vDash(\exists X) \phi(X)$. By our choice of $\phi, \delta \geqslant \gamma$. Hence $\gamma<\omega_{1}^{f}$.

The construction of a tree whose branches "are" models of $K P+V$ $=L+\phi(X)$ which "are" end-extensions of $\delta_{0}$ is straightforward. (The fixed map $g: \omega \cong \delta_{0}$ is used here, and we will arrange that in $M_{f}$ the integer $2 n$ corresponds to $g(n)$.) To insure that $M_{f}$ is well-founded, we have $f$ encode a map $\phi_{f}$ of $O R^{M_{f}}$ into range $(h)$ such that if the given order on $\gamma$ is copied via $h$ onto range $(h), \phi_{f}$ is order preserving. (It is this use of $h$ that causes $T$ to be only $\Sigma_{1}\left(L_{\gamma}\right)$.) This completes our remarks on the construction of $T$.

4.4. Theorem. Let $\sigma$ be the least $\Sigma_{1}^{1}$-reflecting ordinal. Then if $A \in L_{\sigma}, A$ is strongly hyp. encodable.

Proof. We use the known (and fairly easy) inequalities $\sigma<\beta<\beta_{0}$ (cf. [3]). It suffices to see that every ordinal $\gamma$ less than $\sigma$ is strongly hyp. encodable, since if $A \in L_{\sigma}$, there is an ordinal $\gamma<\sigma$ such that $A \leqslant_{h} g$ whenever $\gamma<\omega_{1}^{g}$. But this follows immediately from Lemmas 4.2 and 4.3, via transfinite induction on $\gamma$.

REMARK. Harvey Friedman informs me that he had, some time ago, used the construction of Lemma 4.3 to prove a weaker version of the lemma. Friedman's result appers in Gostanian's N.Y.U. Ph.D. dissertation.

\section{Upper bounds.}

5.1. In this section, we prove that every hyp. encodable set lies in $L_{\sigma}$. We begin by reducing the proof to a pair of lemmas.

We shall presently construct a certain $L_{\sigma}$ ultrafilter, $\mathscr{U}_{\text {. When }}$ we refer to systems of restraint in the following outline, it will always be relative to this fixed ultrafilter $\mathcal{U}_{\text {. Let }} A$ be a fixed subset of $\omega$, with $A \notin L_{\sigma}$.

LEMMA 5.1.1. There is a system of restraints, $R_{0}$, such that whenever $X$ is an infinite subset of $\omega$ satisfying $R_{0}$, then $A \notin L_{\mathrm{o}}[X]$.

LEMMA 5.1.2. There is a system of restraints, $R_{1}$, such that if $X$ is an infinite subset of $\omega$ satisfying $R_{1}$, then there is an infinite subset $Y$ of $X$ such that for every infinite subset $Z$ of $Y, \sigma$ is admissible in $Z$.

Granted these lemmas we show that $A$ is not hyp. encodable. Suppose the 
contrary. Pick $X \subseteq \omega, X$ infinite, such that $X$ satisfies $R_{0}$ and $R_{1}$. Pick $Y$ an infinite subset of $X$ satisfying the conclusions of Lemma 5.1.2. Since $A$ is hyp. encodable, there is an infinite subset $Z$ of $Y$ such that $A \leqslant_{h} Z$.

Since $Y$ satisfies the conclusions of Lemma 5.1.2, $\sigma$ is admissible in $Z$. Since $A \leqslant_{h} Z, A \in L_{\sigma}[Z]$. But $Z$ satisfies $R_{0}$ (since $Z \subseteq X$ ), so $A \notin L_{\sigma}[Z]$, by Lemma 5.1.1. Contradiction!

We have deduced the following theorem from Lemmas 5.1.1 and 5.1.2:

THEOREM. Let $A \subseteq \omega$ be a hyp. encodable set. Then $A \in L_{\sigma}$, where $\sigma$ is the least $\Sigma_{1}^{1}$-reflecting ordinal.

5.2. Before proving Lemmas 5.1.1 and 5.1.2, we must specify the $L_{\sigma}$ ultrafilter $Q$. This we shall now do.

We begin by recalling some properties of $\sigma$ needed in the construction of ${ }_{0}$ All these properties are implicit in [3], and we shall take them as well known. (Proofs of these properties are easy to construct.) First, $\sigma$ is admissible, and is a limit of admissible ordinals. Second, $\sigma$ is projectable into $\omega$. We let $\pi: \sigma \rightarrow \omega$ be a 1-1 map which is $\Sigma_{1}\left(L_{\sigma}\right)$. Finally, if $\alpha<\sigma$, then in $L_{\sigma}, \alpha$ is countable.

The ultrafilter $थ$ that we construct will be $\Delta_{1}\left(L_{\sigma}\right)$. In addition to being selective, it will have the following stronger property which we dub the BorelRamsey property: If $B$ is a Borel subset of $2^{\omega}$ with code $x \in L_{\sigma}$, then there is a $U \in \mathcal{Q}$ which is homogenous for $B$.

Q will also have a property (which is in fact a strengthening of being "decisive") which we shall dub " $\Sigma_{1}^{1}$-decisive". First, we say that a set of integers, $A$, is $थ$-dense if $A \cap U$ is infinite for each $U \in \mathcal{Q}$. There is an evident notion of a $\Sigma_{1}^{1}\left(L_{\sigma}\right)$ property of sets of integers. We say that $\mathcal{Q}$ is $\Sigma_{1^{-}}^{1-}$ decisive if whenever $\psi(\cdot)$ is a $\Sigma_{1}^{1}$ property, and for some 2 -dense $A \subseteq \omega, L_{\sigma}$ $\vDash \psi(A)$, then there is an $A^{\prime} \in \mathcal{U}$ and a $\delta<\sigma$ such that $L_{\delta} \vDash \psi\left(A^{\prime}\right)$. (It is understood that all the parameters appearing in $\psi$ will lie in $L_{\delta}$.)

LEMMA. There is an $L_{\sigma}$ ultrafilter थ with the following properties: (1) थ is selective; (2) थ is Borel-Ramsey; (3) थ is $\Sigma_{1}^{1}$-decisive; (4) थ is $\Delta_{1}\left(L_{o}\right)$.

Proof. It will be useful to adapt the standpoint of meta-recursion theory. That is, we think of $\Delta_{1}\left(L_{\sigma}\right)$ functions as being "effectively computable". We shall frequently talk this way in the present proof.

Broadly speaking, our proof will follow the proof of Lemma 1.2. We shall take steps at successor stages to insure that $थ$ will be Borel-Ramsey. And we shall take steps at certain limit stages to insure that $थ$ is $\Sigma_{1}^{1}$-decisive.

Notice first that since $\sigma$ is an admissible limit of admissibles, given a $\Pi_{1}^{1}$ sentence with real parameters from $L_{\sigma}$, we can effectively determine its truth value. Since the class of codes for Borel sets is $\Pi_{1}^{1}$, we can effectively enumerate the Borel codes in $L_{\sigma}$ in a sequence $\left\langle x_{\eta}: \eta\langle\sigma\rangle\right.$. 
We shall arrange our construction so that $U_{\eta+1}$ is homogenous for the Borel set coded by $x_{\eta}$. In order to do this, we first establish that given $U$ an infinite subset of $\omega$ with $U \in L_{\sigma}, B$ a Borel set with code $x$ in $L_{\sigma}$, there is an infinite subset $V$ of $U$ such that $V \in L_{\sigma}$ and $V$ is homogeneous for $B$. Indeed let $f$ : $\omega \rightarrow U$ enumerate $U$ in increasing order without repetitions. Let

$$
B^{*}=\{A \subseteq \omega \mid f[A] \in B\}
$$

Then $B^{*}$ is a Borel set with code in $L_{\sigma}$. By Corollary 1.10 , there is an infinite set $W$ homogenous for $B^{*}$ with $W \in L_{\sigma}$. We now take $V=f[W]$.

Next, we observe that we can find such a $V$ effectively from $U$ and $x$. Indeed, the question of whether $V \subseteq U$ and $V$ is homogenous for $B$ is $\Pi_{1}^{1}$ in $U$ and $x$ and so can be effectively decided. We now simply search through the subsets of $\omega$ in order of construction until we find such a $V$. It is now clear that we can arrange the construction of $\mathcal{Q}$ so that $U_{\eta+1}$ is homogenous for the Borel set coded by $x_{\eta}$.

It remains to show how to handle the construction at limit stages so as to make थ $\Sigma_{1}^{1}$-decisive. Let $\left\langle\psi_{\eta}(A), \eta\langle\sigma\rangle\right.$ be an enumeration of all $\Sigma_{1}^{1}$ properties of subsets of $\omega$ with parameters in $\sigma$. We may arrange matters so that $\eta$ is larger than any parameters appearing in $\psi_{\eta}$.

Let $\gamma<\sigma$, and let $\psi$ be a $\Sigma_{1}^{1}\left(L_{\gamma}\right)$ statement. Then whether or not $\psi$ is true is effectively decidable in $L_{\sigma}$ since $\gamma$ is countable in $L_{\sigma}$ and usual $\Sigma_{1}^{1}$ statements are effectively decidable. Similarly, if $\psi(A)$ is a $\Sigma_{1}^{1}\left(L_{\gamma}\right)$ property, and $(\exists A) \psi(A)$ is true in $L_{\gamma}$ then we can effectively find an $A$ such that $L_{\gamma} \vDash \psi(A)$ by using the usual basis theorem for $\Sigma_{1}^{1}$ subsets of $P(\omega)$.

Let $\lambda$ be a limit ordinal, and $\eta$ an ordinal $<\lambda$. We say that $\eta$ is attackable at stage $\lambda$ if (1) थ $\cap L_{\lambda}=\left\{U_{\gamma}: \gamma<\lambda\right\}$; (2) There is a $\mathcal{\cap} \cap L_{\lambda}$-dense $A$ such that $L_{\lambda} \vDash \psi_{\eta}(A)$; (3) $\lambda$ is admissible; (4) $\eta$ has not been attacked at some previous stage.

We can effectively determine whether any $\eta<\lambda$ is attackable at stage $\lambda$. If so, we will attack one such $\eta$. For this we use the projection map $\pi: \sigma \rightarrow \omega$, and pick the attackable $\eta$ such that $\pi(\eta)$ is minimal.

We now proceed to discuss the procedure for attacking $\eta$. The statement " $A$ is $\mathcal{Q} \cap L_{\lambda}$-dense and $L_{\lambda} \vDash \psi_{\eta}(A)$ " is $\Sigma_{1}^{1}$. Hence if such an $A$ exists, we can effectively find one. Since $A$ is $\mathscr{U} \cap L_{\lambda}$-dense, we can arrange the choice of $U_{\lambda}$ so that $U_{\lambda} \subseteq A$. This will insure that $A \in \mathcal{Q}$.

The remainder of the details of the construction of $\mathcal{Q}$ are similar to the proof of Lemma 1.2. It remains to see that $थ$ is $\Sigma_{1}^{1}$-decisive. Let $\psi_{\eta}(A)$ be some $\Sigma_{1}^{1}$ property. If for some stage $\lambda, \eta$ is attacked at stage $\lambda$, then there is clearly an $A^{\prime} \in \mathcal{Q}$ such that $L_{\lambda} \vDash \psi_{\eta}\left(A^{\prime}\right)$. Thus, we may assume: (1) There is a थ-dense $A$ such that $L_{\sigma} \vDash \psi_{\eta}(A)$. (2) $\eta$ is never attacked. We shall derive a contradiction. Since $\sigma$ is $\Sigma_{1}^{1}$-reflecting, (1) and (2) imply that $\eta$ is attackable at a cofinal 
set of $\lambda$ 's. Pick $\lambda_{0}<\sigma$ so large that if $\pi\left(\eta^{\prime}\right)<\pi(\eta)$ and $\pi\left(\eta^{\prime}\right)$ is attacked, then it is attacked before stage $\lambda_{0}$. Then if $\eta$ is attackable at stage $\lambda>\lambda_{0}, \eta$ will be attacked, contrary to (2).

We shall fix a $Q$ with the properties stated in the lemma for the remainder of the proof of Theorem 5.1.

5.3. Lemma. Let $F$ be a finite subset of $\omega$. Let $B$ be a Borel set with code in $L_{\sigma}$. Then there is $a U \in Q$ such that (1) $F<U$; (2) for every $A$ such that $F \subseteq A \subseteq F \cup U$,

$$
A \in B \quad \text { iff } \quad F \cup U \in B .
$$

Proof. Let $B^{*}=\{A \subseteq \omega \mid F \cup A \in B\}$. Then $B^{*}$ is Borel with code in $L_{\sigma}$. Pick $U_{1} \in \mathcal{Q}$ homogeneous for $B^{*}$ and let $U=\left\{n>\max (F): n \in U_{1}\right\}$.

5.4. For the proofs of Lemmas 5.1.1 and 5.1.2 we shall adopt the style (though not the substance) of forcing. In particular, let $P$ be $\{\langle F, U\rangle:$ (1) $F$ is a finite subset of $\omega$; (2) $U \in \mathcal{Q}$; (3) $F<U$ \}. We partially order $P$ by saying that $\left\langle F_{0}, U_{0}\right\rangle\left\langle\left\langle F_{1}, U_{1}\right\rangle\right.$ if (1) $F_{1} \subseteq F_{0}$, (2) $U_{0} \subseteq U_{1}$, and (3) $F_{0}-F_{1} \subseteq U_{1}$. We say that $\langle F, U\rangle$ is true of a subset $X$ of $\omega$ if $X$ is infinite and $F \subseteq X \subseteq F \cup U$.

We shall want to study models of the form $L_{\sigma}[X]$, and shall use the usual apparatus of a ramified language with abstraction terms, etc. (Cf. Lévy [9].) There is no trouble handling the syntax of such a language inside $L_{\sigma}$, since $\sigma$ is admissible. (The more elegant approach to forcing of Shoenfield [13] is not suitable since $P$ is a proper class of $L_{\sigma}$.) If $\phi$ is a limited sentence, then $\left\{X \subseteq \omega \mid L_{\sigma}[X] \Vdash \phi\right\}$ is a Borel set with code in $L_{\sigma}$. This is true because, in $L_{\sigma}$, every set is countable. Moreover, we can effectively find such a code from $\phi$.

Let $p \in P$. Let $\phi$ be a limited sentence. We say that $p$ semantically forces $\phi$ (notation: $p \mathbb{I}_{s} \phi$ ) if for every $X$ of which $p$ is true, $L_{o}[X] \Vdash-\phi$. (Caution. This notion differs from that obtained by forcing with the conditions $P$ over the model $L_{\sigma}$. For example, the statement " $X \neq \omega$ " is forced by $\langle\varnothing, \omega\rangle$ but is not semantically forced by $\langle\varnothing, \omega\rangle$.)

Let $p_{i}=\left\langle F_{i}, U_{i}\right\rangle \in P(i=0,1)$. We say that $p_{0}$ is a direct extension of $p_{1}$ if $p_{0} \leqslant p_{1}$ and $F_{0}=F_{1}$.

LEMMA. (1) The relation " $p \Downarrow_{s} \phi$ " is $\Delta_{1}\left(L_{\sigma}\right)$.

(2) Let $\phi$ be a limited sentence. Then every condition $p_{0}$ has a direct extension $p_{1}$ which semantically decides $\phi$. I.e. either $p_{1} \|_{s} \phi$ or $p_{1} \|_{s} \neg \phi$.

(3) Let $p_{1}, p_{2}$ be conditions. If $p_{1}<p_{2}$, and $p_{2} \|_{s} \phi$, then $p_{1} \|_{s} \phi$.

(4) Let $p_{1}, p_{2}$ be conditions which are both direct extensions of $p_{0}$. Then $p_{1}$ and $p_{2}$ are compatible. Hence we cannot have $p_{1} \|_{s} \phi$ and $p_{2} \|_{s} \neg \phi$. 
Proof. (1) Follows from the $\Delta_{1}\left(L_{\sigma}\right)$-decidability of $\Pi_{1}^{1}$ statements. (2) is a rephrasing of Lemma 5.3. (3) follows from the definition of $\|_{s}$, and (4) from the definition of $P$ and the fact that $Q$ is a filter.

5.5. LeMMA. Let $\tau$ be an abstraction term of rank $<\sigma$. Let $F$ be a finite subset of $\omega$. Then there is $a U_{F} \in \mathcal{Q}$ and an $n<\omega$ such that $\left\langle F, U_{F}\right\rangle$ semantically decides " $n \in \tau$ ", and $\left(\left\langle F, U_{F}\right\rangle \Vdash n \in \tau\right)$ iff $n \notin A$. (Thus if $\left\langle F, U_{F}\right\rangle$ is true of $X$, then in $L_{\sigma}[X], \tau$ does not denote $A$.)

PROof. Let $B_{0}$ (resp. $\left.B_{1}\right)=\{n\langle\omega|$ there is an $\langle F, U\rangle \in P$ such that $\langle F, U\rangle \mathbb{I}_{s} n \in \tau$ (resp. $\left.\left.n \notin \tau\right)\right\}$. Then by Lemma 5.4, $B_{1}=\omega-B_{0}$ and each $B_{i}$ is $\Sigma_{1}\left(L_{\sigma}\right)$. Thus, $B_{0}$ is $\Delta_{1}\left(L_{\sigma}\right)$ and hence since $\sigma$ is admissible, $B_{0} \in L_{\sigma}$. Hence, we can find $n$ such that $n \in B_{0}$ iff $n \notin A$. Now select $U_{F} \in \mathcal{Q}$ such that $\left\langle F, U_{F}\right\rangle \in P$ and $\left\langle F, U_{F}\right\rangle$ semantically decides $n \in \tau$.

We can now give the proof of Lemma 5.1.1. Let $\left\langle\tau_{j}, j<\omega\right\rangle$ enumerate the terms of our forcing language. Let $U_{F}$ be chosen for $F, \tau_{i}$ by Lemma 5.5 if $F$ has $i$ elements. Let $R_{0}=\left\{\left\langle F, U_{F}\right\rangle \mid F\right.$ is a finite subset of $\left.\omega\right\}$. Then if $X$ satisfies $R_{0}, A \notin L_{\sigma}[X]$.

RemarK. Suppose that $\alpha$ is the least admissible limit of admissibles. Let $\mathcal{Q}$ be an $L_{\sigma}$ ultrafilter which is selective, Borel-Ramsey, and $\Delta_{1}\left(L_{\alpha}\right)$. (The existence of $थ$ is clear from the easy part of the proof of Lemma 5.2.) Then a variant of Lemma 5.1.1 in which $L_{\sigma}$ is replaced by $L_{\alpha}$ holds for this $\mathcal{Q}$ if $A \notin L_{\alpha}$. It follows that all arithmetically encodable sets lie in $L_{\alpha}$.

5.6. For Lemma 5.1.2, we must insure that $\sigma$ is admissible in $Z$ for certain subsets $Z$ of $X$. Since in $L_{\sigma}$, and hence, in $L_{\sigma}[Z]$, every set is countable, it suffices to insure that every $\Delta_{1}\left(L_{\sigma}[Z]\right)$ map of $\omega$ into $\sigma$, is bounded. Alternatively, let $\left\langle\phi_{i}(x, y, Z), 0\left\langle i\langle\omega\rangle\right.\right.$ be an enumeration of the $\Sigma_{0}$ formulas with parameters in $L_{\sigma}$. Then $\sigma$ will be admissible in $Z$ if for each integer $i$, if $L_{\sigma}[Z] \vDash(\forall n \in \omega)(\exists x) \phi_{i}(x, n, Z)$, then for some $\delta<\sigma$,

$$
L_{\sigma}[Z] \vDash(\forall n \in \omega)\left(\exists x \in L_{\delta}[Z]\right) \phi_{i}(x, n, Z) .
$$

We shall now formulat a "local" analogue of Lemma 5.1.2, which deals with one of the $\phi_{i}$ 's and show that Lemma 5.1.2 follows from the local analogue.

LeMma. Let $\phi(x, y, Z)$ be a $\Sigma_{0}$ formula with parameters in $L_{\sigma}$. Let $F$ be a finite subset of $\omega$. Then there is a system of restraints centered at $F, R_{F}$, with the following property: Let $X$ satisfy $R_{F}$ and let $F$ be an initial segment of $X$. Then there is an infinite subset $Y$ of $X$ with $F \subseteq Y$ such that if $F \subseteq Z \subseteq Y, Z$ is infinite and $L_{\sigma}[Z] \vDash(\forall i \in \omega)(\exists x) \phi(x, i, Z)$ then for some $\delta<\sigma$,

$$
L_{\sigma}[Z] \vDash(\forall i \in \omega)\left(\exists x \in L_{\delta}[Z]\right) \phi(x, i, Z) .
$$

(We express this last by saying $\phi$ is controlled at $Z$.) 
5.7. We now deduce Lemma 5.1.2 from Lemma 5.6. Let $\left\langle\phi_{i}, 0<i<\omega\right\rangle$ be the enumeration of $\Sigma_{0}$ formulas introduced in 5.6.

If $F$ is a finite subset of $\omega$ with $n$ elements, $n>0$, let $R_{F}$ be a system of restraints centered at $F$ obtained by applying Lemma 5.6 to $F$ and $\phi_{n}$. Let

$$
R_{1}=\bigcup\left\{R_{F}: F \text { a finite subset of } \omega\right\} \text {. }
$$

We shall see that $R_{1}$ meets the requirments of Lemma 5.1.2.

Let $X$ be a subset of $\omega$ satisfying $R_{1}$. We construct a set $Y \subseteq X$ meeting the requirments of Lemma 5.1.2.

We are going to construct an infinite sequence of pairs $\left\langle\left\langle F_{n}, X_{n}\right\rangle\right.$; $n\langle\omega\rangle$. We will have: (1) $F_{n}$ has $n$ elements, $X_{n}$ is infinite, and $F_{n}<X_{n}$; (2) $X_{0}=X$; (3) $F_{n} \subseteq F_{n+1} \subseteq F_{n} \cup X_{n}$; (4) $X_{n+1} \subseteq X_{n}$; (5) let $k>0$. Let $G \subseteq F_{n}$ have $k$ elements. Let $G \subseteq Z \subseteq G \cup X_{n}$. Then $\phi_{k}$ is controlled at $Z$.

Suppose we can construct $\left\langle F_{n}, X_{n}\right\rangle$ satisfying (1)-(5). Let $Y=\cup\left\{F_{n}: n\right.$ $\in \omega$. Let $Z$ be an infinite subset of $Y$. Then we show that $\sigma$ is admissible in $Z$. For this it is enough to see that each $\phi_{k}$ is controlled at $Z$.

Fix $k>0$. Let $G$ be the first $k$ elements of $Z$. Pick $n$ minimal such that $G \subseteq F_{n}$. Then $Z \subseteq F_{n} \cup X_{n}$ and hence by (5), $\phi_{k}$ is controlled at $Z$.

It remains to construct the sequence $\left\langle F_{n}, X_{n}\right\rangle$ satisfying (1)-(5). If $n=0$, put $F_{0}=\varnothing, X_{0}=X$. Let $n=k+1$. Let $m$ be the least element of $X_{k}$. Set $F_{k+1}=F_{k} \cup\{m\}$. Let $G_{1}, \ldots, G_{N}$ enumerate the nonempty finite subsets of $F_{n+1}$. We shall define a decreasing sequence of infinite sets

$$
X_{k} \supset Y_{0} \supseteq Y_{1} \supseteq \cdots \supseteq Y_{N},
$$

so that (1) $F_{n+1}<Y_{0}$; (2) if $G_{l} \subseteq Z \subseteq G_{l} \cup Y_{l}$, and $G_{l}$ has $r$ elements, then $\phi_{r}$ is controlled at $Z$. If we can do this, we may take $X_{k+1}=Y_{N}$ and satisfy (1)-(5).

We take $Y_{0}=X_{n} / F_{n+1}$. Suppose $1 \leqslant l \leqslant N$, and $G_{l}$ has $r$ elements. Since $G_{l} \cup Y_{l-1} \subseteq X, G_{l} \cup Y_{l-1}$ satisfies $R_{G_{l}}$. Hence by Lemma 5.6 (and our choice of $R_{G_{l}}$ ) we may choose $Y_{l} \subseteq Y_{l-1}$ as desired in the preceding paragraph.

This completes our reduction of Lemma 5.1.2 to 5.6.

5.8. Our proof of Lemma 5.6 is complicated and we now outline the main steps.

(1) We construct a system of restraints $R_{0}$ such that if $X$ satisfies $R_{0}$, and a limited sentence, $\phi$, holds in $L_{\sigma}[X]$, then $\phi$ is semantically forced by some condition $p \in P$ which holds for $X$.

(2) We then study the situation where $F$ is a finite subset of $\omega$, and $\phi(x, X)$ is a $\Sigma_{0}$ formula with parameters in $L_{\sigma}$. We show that either (a) for some $U \in \mathcal{U}$, and some $\delta<\sigma$,

$$
\langle F, U\rangle \mathbb{I}_{s}\left(\exists x \in L_{\delta}[X]\right) \phi(x, X)
$$


or (b) there is a system of restraints, $R$, centered at $F$, such that if $F$ is an initial subset of $X$ and $X$ satisfies $R$, then

$$
L_{\sigma}[X] \vDash(\forall x) \neg \phi(x, X) .
$$

(3) We then turn to the specific situation of Lemma 5.6. In this outline, we shall discuss the special case when the $F$ of $\$ 5.6$ is $\varnothing$.

Let $G$ be a finite set with $k$ elements. We say that $\phi$ is bounded at $G$ if alternative (a) of (2) holds for $G$ and $\phi(\cdot, k, \cdot)$.

Suppose there is a $U \in \mathcal{Q}$ such that $\phi$ is bounded at each finite subset $G$ of $U$. Then it is not hard to show that $\langle\phi, U\rangle$ semantically forces $(\forall i \in \omega)$ $\left(\exists x \in L_{\delta}[X]\right) \phi(x, i, X)$ for some $\delta<\sigma$. So in this case, Lemma 5.6 is clear.

(4) Because $O$ is $\Sigma_{1}^{1}$-decisive, the situation of the preceding paragraph tends to hold. If it fails, we will be able to construct a system of restraints $R^{\prime}$, such that if $X$ satisfies $R^{\prime}$, then there is an infinite subset $Y$ of $X$ such that

$$
L_{\sigma}[Y] \vDash(\exists i \in \omega)(\forall x) \neg \phi(x, i, Y) .
$$

(5) The conclusion of (4) is not strong enough for Lemma 5.6. However, using the Galvin-Prikry theorem for open sets, we will be able to conclude that there is a $Y \subseteq X, Y$ infinite, such that for all $Z \subseteq Y, Z$ infinite, we have:

$$
L_{\sigma}[Z] \vDash(\exists i \in \omega)(\forall x) \neg \phi(x, i, Y) .
$$

So Lemma 5.6 will hold in this case also. This completes our outline.

5.9. Definition. $X$ is $s-थ$ generic over $L_{\sigma}$ if (1) $X$ is an infinite subset of $\omega$; (2) whenever $L_{\sigma}[X] \vDash \phi$, for $\phi$ a limited sentence, then $p \mathbb{r}_{s} \phi$ for some $p \in P$ true of $X$.

Lemma. Let $F$ be a finite subset of $\omega$. Then there is a system of restraints centered at $F, R_{F}$, such that if $X$ satisfies $R_{F}$, and $X$ is an end-extension of $F$, then $X$ is $s-$ Q generic over $L_{\sigma}$.

Proof. Let $\left\langle\phi_{i}, i<\omega\right\rangle$ be an enumeration of the limited sentences in which each one appears infinitely of ten. Let $G$ be a finite subset of $\omega$ with $n$ elements. Pick $U_{G} \in \mathcal{Q}$ so that $\left\langle G, U_{G}\right\rangle$ semantically decides $\phi_{n}$ (by Lemma 5.4.2). Take $R_{F}=\left\{\left\langle G, U_{G}\right\rangle: G\right.$ is a finite end-extension of $\left.F\right\}$.

5.10. Lemma. U is decisive.

Proof. Let $A=\{n \in \omega \mid \psi(n)\}$ where $\psi$ is $\Sigma_{1}$. Suppose that $A$ is Q-dense. We show that $U \subseteq A$ for some $U \in \mathcal{Q}$. Consider the property $\theta(X)$ which states that $X$ is $\{n \in \omega \mid \psi(n)\}$. Then $\theta$ is $\Sigma_{1}^{1}$, and $\theta(A)$ holds for some Q parameters in $\theta$ appear in $L_{\delta}$, and an $A_{\delta} \in \mathcal{Q}$ such that $A_{\delta}=\left\{n \in \omega \mid L_{\delta}\right.$ $\vDash \psi(n)\}$. But since $\psi$ is $\Sigma_{1}, A_{\delta} \subseteq A$. 
5.11. We now let $\phi(x, X)$ be a fixed $\Sigma_{0}$ formula with parameters from $L_{\sigma}$. If $F$ is a finite subset of $\omega$, we say that $\phi$ is bounded at $F$ if there is a $U \in \mathcal{Q}$ and a $\delta<\sigma$ such that $\langle F, U\rangle=p \in P$, and $p \mathbb{v}_{s}\left(\exists x \in L_{\delta}(X)\right.$ $\phi(x, X))$.

Lemma. Suppose that $F$ is a finite subset of $\omega$, and that $\phi$ is not bounded at $F$. Then there is a $U \in \mathcal{Q}$ such that for each $n \in U, \phi$ is not bounded at $F \cup\{n\}$.

PRoof. $\{n \in \omega \mid \phi$ is bounded at $F \cup\{n\}\}$ is clearly $\Sigma_{1}\left(L_{\sigma}\right)$. Since $थ$ is decisive (by Lemma 5.10), if the lemma fails there is a $U \in \mathcal{Q}$ such that $\phi$ is bounded at $F \cup\{n\}$ for each $n \in U$. We assume this and proceed to show that $\phi$ is bounded at $F$, contrary to our assumptions.

By $\Sigma_{1}$ choice, there is a function $g\left(=\left\langle\left\langle\delta_{n}, U_{n}\right\rangle: n \in U\right\rangle\right)$ in $L_{\sigma}$ such that

$$
\left\langle F \cup\{n\}, U_{n}\right\rangle \Vdash_{s}\left(\exists x \in L_{\delta_{n}}\right) \phi(x, X),
$$

for each $n \in U$. Let $\delta=\max \left\{\delta_{n}: n \in U\right\}$. Then $\delta<\sigma$ since $g \in L_{\sigma}$. Since Q is selective, we can find $V \in \mathcal{Q}$ such that (1) $V \subseteq U$; (2) if $n \in V, V /\{n\}$ $\subseteq U_{n}$. Then clearly $\langle F, V\rangle \mathbb{I}_{s}\left(\exists x \in L_{\delta}[X]\right) \phi(x, X)$.

5.12. Lemma. Let $\phi(x, X)$ be a $\Sigma_{0}$ formula with parameters in $L_{\sigma}$. Let $F$ be a finite subset of $\omega$. Then either (a) there is $a U \in \mathcal{U}$ and $a \delta<\sigma$ sucil that

$$
\langle F, U\rangle \mathbb{R}_{s}\left(\exists x \in L_{\delta}[X]\right) \phi(x, X)
$$

or (b) there is a system of restraints centered at $F, R_{F}$, such that if $X$ is an endextension of $F$ satisfying $R_{F}$, then

$$
L_{\sigma}[X] \vDash(\forall x) \neg \phi(x, X) .
$$

Proof. Assume that alternative (a) fails. Then $\phi$ is not bounded at $F$ in the sense of \$5.1.1. For each finite subset $G$ of $\omega$ such that $\phi$ is not bounded at $G$, select $U_{G}$ by Lemma 5.11. Let $R_{0}=\left\{\left\langle G, U_{G}\right\rangle: G\right.$ is a finite end-extension of $F$ and $\phi$ is not bounded at $G$ \}. Then if $X$ is an end-extension of $F$ and $X$ satisfies $R_{0}$, then if $G$ is a finite initial segment of $X$ and $F \subseteq G$, then $\phi$ is not bounded at $G$. (The proof is by induction on the size of $G$.)

Let $R_{1}$ be a system of restraints centered at $F$ given by Lemma 5.9. Let $R=R_{0} \cup R_{1}$. We show that if $X$ is an end-extension of $F$ satisfying $R$, then $(\forall x) \neg \phi(x, X)$ holds in $L_{\mathrm{o}}[X]$.

Suppose not. Then for some $\delta<\sigma$, we have $L_{\sigma}[X] \vDash\left(\exists x \in L_{\delta}[X] \phi(x, X)\right)$. Since $X$ satisfies $R_{1}$, we can find a condition $\langle G, U\rangle$ true of $X$ such that $\langle G, U\rangle \mathbb{\Downarrow}_{s}\left(\exists x \in L_{\delta}[X]\right) \phi(x, X)$. Without loss of generality (by Lemma 5.4) we may assume $F \subseteq G$. Hence $\phi$ is bounded at $G$ with $F \subseteq G \subseteq X$ contrary to $X$ being an end-extension of $F$ satisfying $R_{0}$. 
5.13. We turn now to the proof of Lemma 5.6. We fix a finite set $F$ and a $\Sigma_{0}$ formula $\phi(x, y, X)$ with parameters from $L_{\sigma}$. If $G$ is a finite end-extension of $F$, let $i(G)$ be the number of elements in $G-F$. We say that $\phi$ is bounded at $G$ if the formula $\phi^{\prime}(x, X)=\phi(x, i(G), X)$ is bounded at $G$ in the sense of $\$ 5.11$.

LEMMA. Suppose that for some $U \in \mathcal{U}$, the following hold: (1) $F<U$; (2) for each finite $G$ such that $F \subseteq G \subseteq F \cup U, \phi$ is bounded at $G$. Then there is $a$ $\delta<\sigma$ and $a V \in \mathcal{Q}$ such that $\langle F, V\rangle \in P$, and

$$
\langle F, V\rangle \mathbb{I}_{s}(\forall i \in \omega)\left(\exists x \in L_{\delta}[X]\right) \phi(x, i, X) .
$$

Hence Lemma 5.6 holds for $F$ and $\phi$.

Proof. The proof is quite similar to that of Lemma 5.11.

5.14. From now on, we assume that no $U$ satisfies the hypothesis of Lemma 5.13 for our particular choice of $F$ and $\phi$.

LEMMA. Let $A$ be a $थ$-dense subset of $\omega$. Suppose that $F$ is an initial segment of $A$. Then there is a finite subset $G$ of $A$ such that $F \subseteq G$ and $\phi$ is not bounded at $G$.

Proof. Our plan is to deny the lemma and use the $\Sigma_{1}^{1}$-decisiveness of $\mathscr{U}$ to show that the hypotheses of 5.13 are satisfied contrary to our assumption.

The property denied for $A$ by the lemma is clearly $\Sigma_{1}^{1}$. Hence if it holds for some $थ$-dense $A$, then for some $A \in \mathcal{Q}$ and some $\delta<\sigma$, we have: (1) $F$ is an initial segment of $A$; (2) $\delta$ is an admissible limit of admissibles; (3) the same $\Delta_{1}$ definition that defines $थ$ in $L_{\sigma}$ defines $थ \cap L_{\delta}$ in $L_{\delta}$; (4) for every finite subset $G$ of $A$ with $F \subseteq G, \phi$ is bounded (in the sense of $L_{\delta}$ ) at $G$.

The key point is that if $\phi$ is bounded at $G$ (in the sense of $L_{\delta}$ ) then a fortiori, $\phi$ is bounded at $G$ (in the sense of $L_{\sigma}$ ). Hence $A / F$ satisfies the hypotheses of Lemma 5.13.

5.15. We now define an open set $W$, and a system of restraints $R$. First, let $W=\{A \subseteq \omega \mid F<A$ and for some finite initial segment $H$ of $A, \phi$ is not bounded at $F \cup H\}$.

The definition of $R$ is a little more involved. First, if $G$ is a finite endextension of $F$, and $\phi$ is not bounded at $G$, we let $R_{G}$ be a system of restraints, given by Lemma 5.12 which is centered at $G$ and such that if $X$ is an endextension of $G$ satisfying $R_{G}$, then

$$
L_{\sigma}[X] \vDash(\forall x) \neg \phi(x, i(G), X) .
$$

Let $R^{*}$ be a system of restraints centered at $F$ such that if $X$ satisfies $R^{*}$ and $X$ is an end-extension of $F$, then $X$ is $थ$-dense $\left(R^{*}\right.$ is easy to construct since $Q$ is countable). Let $R=R^{*} \cup\left\{R_{G}: G\right.$ is an end-extension of $\left.F\right\}$. The crucial properties of $R$ and $W$ are summed up in the following lemma. 
Lemma. Let $X$ be an end-extension of $F$ satisfying $R$. Then if $X / F$ lies in $W$, then $L_{\sigma}[X] \vDash(\exists i)(\forall x) \neg \phi(x, i, X)$. Moreover, if $X$ is an end-extension of $F$ satisfying $R$, then $X$ has an infinite subset $Y$ with $F \subseteq Y$ such that $Y / F \in W$.

Proof. If $X / F$ lies in $W$, then for some initial segment $H$ of $X / F, \phi$ is not bounded at $F \cup H$. Set $G=F \cup H$, and let $n=i(G)$. Since $X$ satisfies $R$, it also satisfies $R_{G}$. Since $G$ is an initial segment of $X, L_{\sigma}[X] \vDash(\forall x) \neg \phi(x, n, X)$. This proves our first claim.

For the second, note that $X$ is थ-dense. Hence by Lemma 5.14, we may find a finite subset $H$ of $X / F$ such that $\phi$ is not bounded at $F \cup H$. Let $Y$ be an infinite subset of $X$ such that $F \cup H$ is an initial segment of $Y$. Then clearly $F \subseteq Y \subseteq X$, and $Y / F \in W$.

5.16. We are now in a position to complete the proof of Lemma 5.6. If the hypotheses of Lemma 5.13 hold, then we are done by Lemma 5.13. If not, we show that the $R$ constructed in 5.15 meets the demands of $\$ 5.6$. Let then $X$ be an end-extension of $F$ satisfying $R$. By the Galvin-Prikry theorem, select $A \subseteq X / F$ homogeneous for $W$. From the second half of Lemma 5.15, we see that $A$ lands in $W$.

Now let $Y=A \cup F$. If $F \subseteq Z \subseteq Y$, and $Z$ is infinite, then $Z$ meets $R$, since $X$ does. Moreover, since $A$ lands in $W, Z / F \in W$. Hence by the first half of Lemma 5.15,

$$
L_{\sigma}[Z] \vDash(\exists i)(\forall x) \neg \phi(x, i, Z) .
$$

This completes our proof of Lemma 5.6, and with it, the proof of Theorem 5.1.

6. Some conjectures.

6.1. Our results can be interpreted in such a way as to have obvious generalizations to the higher levels of the projective hierarchy. I shall make these interpretations (which are clear to anyone familiar with descriptive set theory and nonmonotone inductive definitions) and then formulate the generalizations as conjectures. I have not thought much about these conjectures and they may have fairly easy refutations or proofs.

6.2. First, here is the characterization of $\sigma$ that relates it to descriptive set theory: According to Aczel-Richter [3], $\sigma$ is the closure ordinal of $\Sigma_{1}^{1}$ nonmonotone definitions. (In their notation, $\sigma=\left|\Sigma_{1}^{1}\right|$.)

Suppose now that $n>1$. The analogue of $\sigma$ will be one of $\left|\Sigma_{n}^{1}\right|$ and $\left|\Pi_{n}^{1}\right|$. But which one should we pick? Recall the following result of Aanderra [1]. If pre-well-ordering holds for $\Sigma_{n}^{1}\left(\Pi_{n}^{1}\right)$ then $\left|\Pi_{n}^{1}\right|>\left|\Sigma_{n}^{1}\right|$ (resp. $\left.\left|\Sigma_{n}^{1}\right|>\left|\Pi_{n}^{1}\right|\right)$.

The reader should interpret our conjectures as predictions for the theory $\mathrm{ZFC}+\mathrm{PD}\left({ }^{5}\right)$.

(5) Projective Determinacy. 
(1) The notions " $\Delta_{n}^{1}$-encodable" and "strongly $\Delta_{n}^{1}$-encodable" are coextensive (cf. §4.1).

(2) Let $\gamma_{n}=\max \left(\left|\Sigma_{n}^{1}\right|,\left|\Pi_{n}^{1}\right|\right)$. Then an ordinal $\gamma$ is $\Delta_{n}^{1}$-encodable (cf. $\left.\$ 4.2\right)$ iff $\gamma<\gamma_{n}$.

Now let PD hold. Let $n^{*}=n$ if $n$ is odd, and let $n^{*}=n-1$ for $n$ even. Let $\mathcal{C}$ be the maximal countable $\Pi_{n^{*}}^{1}$ set. According to Kechris [8], $\mathcal{C}$ is a union of a well-ordered sequence of $\Delta_{n^{*}}^{1}$ degrees, $\left\langle d_{\xi}\right| \xi\left\langle\lambda_{n^{*}}\right\rangle$. Moreover, it is trivial to see that $\gamma_{n}<\lambda_{n^{*}}$. We conjecture that the $\Delta_{n}^{1}$-encodable sets are precisely those sets $\Delta_{n}^{1}$ in some $d_{\xi}$ for $\xi<\gamma_{n}$. Note that if $V=L$, every set of integers is $\Delta_{2}^{1}$-encodable. This follows easily from the fact that, in $L$, the $\Delta_{2}^{1}$ degrees are well ordered of order type $\aleph_{1}$.

\title{
BIBLIOGRAPHY
}

1. S. Aanderra, Inductive definitions and their closure ordinals, Generalized Recursion Theory (J. E. Fenstad and P. G. Hinman, eds.), North-Holland, Amsterdam, 1974.

2. P. Aczel, Representability in some systems of second order arithmetic, Israel J. Math. 8 (1970), 309-328. MR 42 \# 4396.

3. P. Aczel and W. Richter, Inductive definitions and analogues of large cardinals (Conference in Mathematical Logic-London '70), Lecture Notes in Math., vol. 225, Springer-Verlag, Berlin and New York, 1972, pp. 1-9.

4. F. Galvin and K. Prikry, Borel sets and Ramsey's theorem, J. Symbolic Logic 38 (1973), 193-198. MR 49 \#2399.

5. R. B. Jensen, The fine structure of the constructible hierarchy, Ann. Math. Logic 4 (1972), 229-308; erratum, ibid. 4 (1972), 443. MR 46 \#8834.

6. C. G. Jockusch, Jr., Uniformly introreducible sets, J. Symbolic Logic 33 (1968), 521-536. MR 38 \#5619.

7. C. G. Jockusch, Jr. and R. I. Soare, Encodability of Kleene's O, J. Symbolic Logic 38 (1973), 437-440.

8. A. Kechris, The theory of countable analytical sets, Trans. Amer. Math. Soc. 202 (1975), 259-297.

9. Azriel Lévy, Definability in axiomatic set theory. I, Logic, Methodology, and Philos. Sci. (Proc. 1964 Internat. Congr.), North-Holland, Amsterdam, 1965, pp. 127-151. MR 34 \#5653.

10. A. R. D. Mathias, On a generalization of Ramsey's theorem, Notices Amer. Math. Soc. 15 (1968), 931. Abstract \#68T-E19; Lecture Notes in Math., Springer-Verlag, Berlin (to appear).

11. H. Rogers, Jr., Theory of recursive functions and effective computability, McGraw-Hill, New York, 1967. MR 37 \#61.

12. G. Sacks and S. Simpson, The $\alpha$-finite injury method, Ann. Math. Logic 4 (1972), 343-367.

13. J. R. Shoenfield, Unramified forcing, Proc. Sympos. Pure Math.,vol.13, part 1, Amer. Math. Soc., Providence, R. I., 1971, pp. 357-381. MR 43 \#6079.

14. R. Soare, Sets with no subset of higher degree, J. Symbolic Logic 34 (1969), 53-56. MR 41 $\# 8228$.

15. R. Solovay, A model of set-theory in which every set of reals is Lebesgue measurable, Ann. of Math. (2) 92 (1970), 1-56. MR 42 \#64.

\author{
Mathematical Sciences Department, IBM Corporation, Yorktown Heights, New York \\ 10598
}

Current address: Department of Mathematics, University of California, Berkeley, California 94720. 